\title{
Assessing gestational age of babies: Performance of obstetric ultrasound scan compared to that from the combination of Naegle's rule and Dubowitz score in the 21st century
}

\author{
Baba Usman Ahmadu \\ Department of Paediatrics, Federal Medical Centre, Yola, Nigeria; ahmadu4u2003@yahoo.com
}

Received 13 June 2013; revised 13 July 2013; accepted 20 July 2013

Copyright (C) 2013 Baba Usman Ahmadu. This is an open access article distributed under the Creative Commons Attribution License, which permits unrestricted use, distribution, and reproduction in any medium, provided the original work is properly cited.

\section{ABSTRACT}

Background: Some researchers have suggested that when Naegle's rule (NR) and Dubowitz score (DS) are combined, it could out-perform obstetric ultrasound scan (USS). Others still believe that obstetric USS alone is still effective relative to the combination of NR rule and DS in assessing the gestational age (GA) of babies. Objectives: To determine and compare the GA of babies using obstetric USS, NR and DS; and to provide relevant public health information on obstetric USS in the 21st century. Methods: Subjects were selected using systematic random sampling and the GA of babies was determined using obstetric USS, NR, and DS. Statistical package for social science (SPSS) statistical software version 16, Illinois, Chicago USA was used for all data analysis. Results: Eightyfive mother-baby pairs were studied. Forty-four babies (52\%) were males and $41(48 \%)$ females. Sixty four (75\%) were term with a mean (SD) BW of $3.02(0.59)$ at $95 \% \mathrm{Cl}(2.89-3.14) \mathrm{kg}$. The overall mean GA of the babies was $38.49(2.89)$ at $95 \% \mathrm{Cl}(38.14$ - 38.85) weeks. The mean GA using obstetric USS, NR and DS were 38.52 (1.98) at $95 \% \mathrm{Cl}(38.14$ - 38.99), $38.09(4.13)$ at $95 \% \mathrm{Cl}$ (37.20 - 38.99) and $38.82(2.02)$ at $95 \% \mathrm{Cl}(38.39$ 39.26), but comparison of these means was not significant $(p=0.256)$. Combined mean $G A$ by NR and DS was $38.46(3.26)$ at $95 \% \mathrm{Cl}$ (37.96 38.95). Comparing this mean with mean GA obtained by obstetric USS was also not significant $(p=0.885)$. Conclusion: The GA assessments by Obstetric USS, NR and DS were all reliable, and Obstetric USS performed effectively relative to combined NR and DS.

Keywords: Gestational Age; Babies; Obstetric Ultrasound Scan; Naegle's Rule; Dubowitz Score; Maiduguri; Nigeria

\section{INTRODUCTION}

To a neonatologist, the gestational age (GA) of babies provides important information on maturity and survival from birth onwards. For a long time, obstetric ultrasound scan (USS), Dubowitz score (DS) and Naegle's rule (NR) are being used to determine the GA of babies [1-3]. Not one of these is without a drawback when used alone; based on this, many authors have advocated combining these methods to assess the GA of babies [1-3]. The use of DS for instance requires technical know-how and maternal stress leading to premature neurological maturation could obscure the DS [1]. Sunjoh et al. [4], in 2004 in the Cameroon however argued that the DS could still be accurate even in maternal stressful conditions. Wingate et al. [5], in 2007 has challenged the validity and also the accuracy of NR, with reason being the tendency to overestimate GA because mothers may not remember their last menstrual period (LMP). Overestimating the GA of a baby would lead to falsely high number of post-date babies and little of preterm deliveries. A previous study has reported that the NR is more important for gynaecologic rather than for paediatric use [1]. This is due to its abortion safety if applied correctly early in pregnancy.

Before now, obstetric USS has been found to be more useful in assessing the GA of a foetus during early trimesters of pregnancies even though the NR, which uses the LMP may be preferable if LMP is accurate [1-3]. Because mothers are not likely to be accurate regarding 
dates and regularity of their cycle, obstetric USS may be a better alternative in assessing the GA of foetuses early in pregnancy. Many investigators had found that, combining NR and DS to assess the GA of babies may be more reliable than obstetric USS [1,6,7]. In this light, this study aimed at: 1) to determine the GA of babies using obstetric USS, DS and NR; 2) to compare the mean GA of the babies obtained by obstetric USS, DS and NR; 3) to provide relevant public health information on the importance of obstetric USS in the $21 \mathrm{st}$ century. To my knowledge, no such study has been conducted in the North-East sub-region of Nigeria and little is also known of this subject in developed countries after extensive literature search.

\section{SUBJECTS AND METHODS}

\subsection{Study Site}

The study was conducted at the Department of Paediatrics and Obstetrics unit of the University of Maiduguri Teaching Hospital, (UMTH), Borno State, Nigeria. Maiduguri is a cosmopolitan city situated in the North-Eastern part of Nigeria and the UMTH is the largest tertiary health institution situated in the region. The UMTH serves as a referral hospital for six North-Eastern States of Nigeria and neighboring countries of Chad, Cameroon and Niger Republics.

\subsection{Ethics}

Ethical clearance was obtained from the Ethics Committee of the UMTH. Informed consent was obtained from parents after explaining the purpose and the objectives of the study. Parents had unlimited liberty to deny consent or opt out of the study without any consequences. All work was performed according to the international guidelines for human experimentation in clinical research [8].

\subsection{Sample Size/Subject Selection}

The minimum sample size was determined using statistical formula, which computes five percent fertility rate for Nigeria at $95 \%$ confidence interval and alpha levels of $0.05[9,10]$. This sum up to 77 ; but $10 \%$ of this was added to maximize power. Therefore, the study population consisted of 85 mother-baby pairs. Participation in this study was voluntary and consenting pregnant women were selected using systematic random sampling method where the first of every five pregnant woman was picked as they presented to the labor ward. Where the first did not fulfill the inclusion criteria, the immediate next pregnant woman that qualified was selected.

\subsection{Inclusion and Exclusion Criteria}

Pregnant women who had antenatal care and delivered well babies at the UMTH were eligible to participate in this study. Pregnant women who smoke cigarettes or are severely sick or those who decline consent for the study were excluded.

\subsection{Procedure}

The GA of babies using NR and obstetric USS at term was obtained from maternal antenatal health care records using a questionnaire, and the DS was used to access the GA of babies at birth $[2,11,12]$. Birth weight (BW) of the babies was measured using the basinet weighing scale that has a sensitivity of 50 grams. Babies with GA less than 37 completed weeks were classified as preterm, those from 37 completed to less than 42 completed weeks were term, and those with 42 completed weeks or more were post term [13].

\subsection{Data Analysis}

Data were expressed as frequencies; mean (SD) and 95\% confidence intervals (CIs). Analysis of variance (ANOVA) and Student $t$ test were used to investigate quantitative variables. A p-value $<0.05$ was considered significant. Tables were used for demonstrations. Statistical package for social science (SPSS) statistical software version 16, Illinois, Chicago USA was used for all analysis.

\section{RESULTS}

A total of 85 mother-baby pairs were studied. There were $44(52 \%)$ males and $41(48 \%)$ females giving a male-female ratio of 1.07:1 (Table 1). Most babies were term $64(75 \%)$ and mean (SD) BW was $3.02(0.59)$ at $95 \%$ CI $(2.89-3.14) \mathrm{kg}$.

The overall mean GA of the babies was 38.49 (2.89) at 95\%CI (38.14 - 38.85) weeks. Table 2 shows the mean GA distribution of the babies using obstetric USS, NR and DS. The comparison of mean GA of the babies using obstetric USS, NR and DS was not significant ( $\mathrm{p}=$ 0.256). When the GA obtained by NR and DS were combined, the mean GA for the combination was 38.46 (3.26) at $95 \%$ CI $(37.96$ - 38.95). Comparing this mean with the mean GA from obstetric USS was also not significant $(\mathrm{p}=0.885)$ (Table 3$)$.

Table 1. Gestational age and sex distribution of the babies.

\begin{tabular}{cccc}
\hline GA (Weeks) & Male & Female & Total \\
\hline Preterm $(<37)$ & 4 & 5 & 9 \\
Term $(\geq 37<42)$ & 32 & 32 & 64 \\
Post term $(\geq 42)$ & 8 & 4 & 12 \\
Total & 44 & 41 & 85 \\
\hline
\end{tabular}

$\mathrm{GA}=$ Gestational age. 
Table 2. Mean gestational age of the babies using obstetric ultrasound scan, Naegle's rule and Dubowitz score.

\begin{tabular}{lll}
\hline \multirow{2}{*}{ Parameters } & \multicolumn{2}{c}{ Gestational age (weeks) } \\
& Mean (SD) & 95\%CIs \\
\hline Obstetric USS & $38.52(1.98)$ & $38.14-38.99$ \\
NR & $38.09(4.13)$ & $37.20-38.99$ \\
DS & $38.82(2.02)$ & $38.39-39.26$ \\
p value $^{\mathrm{a}}$ & 0.256 & - \\
\hline
\end{tabular}

USS = Ultrasound scan, NR = Naegle's rule, DS = Dubowitz score, CIs = Confidence intervals; $p$ value ${ }^{a}=$ Analysis of variance (ANOVA).

Table 3. Comparison of mean gestational age of babies by obstetric ultrasound scan and combined Naegle's rule and Dubowitz score.

\begin{tabular}{lll}
\hline \multirow{2}{*}{ Parameters } & \multicolumn{2}{c}{ Gestational age (weeks) } \\
& Mean (SD) & 95\%CIs \\
\hline Obstetric USS & $38.52(1.98)$ & $38.14-38.99$ \\
Combine (NR and DS) & $38.46(3.26)$ & $37.96-38.95$ \\
p value $^{\mathrm{b}}$ & 0.885 & - \\
\hline
\end{tabular}

USS $=$ Ultrasound scan, $\mathrm{NR}=$ Naegle's rule, $\mathrm{DS}=$ Dubowitz score, $\mathrm{CIs}=$ Confidence intervals; $p$ value ${ }^{\mathrm{b}}=$ Student $\mathrm{t}$ test.

\section{DISCUSSIONS}

Obstetric USS, NR and DS all performed effectively in current study. Similar finding was observed by Mitchell [2] in Guy's Hospital, London in 1979. The most likely explanation for this observation could be the correctness of maternal LMP, technical know-how in doing obstetric USS and DS. In Nigeria, the adult literacy level and female education especially on reproductive health is high, this might be the reason most of the mothers were able to state accurately their LMP [10]. More so, mothers that participated in this work had their antenatal care supervised by qualified gynaecologist who at intervals performed obstetric USS. Babies delivered to these mothers had their DS also administered by qualified medical personnel. In a population where literacy levels are low, LMP can be unreliable and in such situations, the DS which is simple and inexpensive unlike obstetric USS can be used [3].

Of note, however, is that the obstetric USS remains most preferable for estimating the GA of sick babies, example, perinatal asphyxia, a condition which could limit the validity of DS [2]. Furthermore, female babies in some reports gave conflicting findings. Whereas, Parkin et al., [14] in 1976 found no sex related differences in GA when conducted by obstetric USS, Henriksen [3], in 1995 published that female sex can interfere negatively with obstetric USS. This is because the female sex is a factor that reduces the size of a foetus and that could falsely lower the GA of a foetus.
Some researchers have suggested that when NR is combined with DS to assess the GA of babies, the performance may be more reliable than that of obstetric USS alone [1,6,7]. This, however, was not observed in this study possibly because of technological advancement build in USS machine of the 21 st century. The DS determines postnatal age of babies, Obstetric USS basically determines antenatal GA, whereas NR could assessed either antenatal or postnatal age. Prenatal and postnatal estimates of GA combined together would be more appropriate based on rational thinking. This combination was, however, inconsistent because GA could either be underestimated or overestimated $[1,6,7]$. The NR depends upon maternal knowledge of LMP and regular 28-day cycle. Certain biological differences like extremes of age, multiparity and negroid race have been associated with shorter LMP cycles, whereas, white and age group 19 to 34 years have been linked to longer LMP [15]. These might be the cause of the inconsistencies that were observed; as such, adequate maternal history is paramount in assessing GA of babies by NR alone or in combination with DS.

\section{CONCLUSION}

The GA assessment by Obstetric USS compared well with that of NR and DS in present study. More so, Obstetric USS performed effectively in determining the GA of the babies relative to the combined NR and DS.

\section{LIMITATION}

The sample size used could constitute a setback to this work. Larger sample size would have been more appropriate in order to generalize these findings.

\section{RECOMMENDATIONS}

For a more reliable determination of GA of babies, Obstetric USS is hereby recommended and health managers should provide this machine especially at the maternity units of hospitals for quality neonatal care. There is also the need for larger population sample for studies of this kind in the future in order to improve on the validity of results.

\section{ACKNOWLEDGEMENTS}

I thank all the medical, paramedical and administrative staff of the UMTH for their help and support.

\section{REFERENCES}

[1] Rosenberg, R.E., Ahmed, N.U., Ahmed, S., Saha, S.K., Chowdhury, A., Black, R.E., et al. (2009) Determining gestational age in a low-resource setting: Validity of last menstrual period. Journal of Health, Population and $\mathrm{Nu}$ - 
trition, 27, 332-338.

[2] Mitchell, D. (1979) Accuracy of pre and postnatal assessment of gestational age. Archives of Disease in Childhood, 54, 896-904. doi:10.1136/adc.54.11.896

[3] Henriksen, T.B., Wilcox, A.J., Hedegaard, M. and Secher, N.J. (1995) Bias in studies of preterm and postterm delivery due to ultrasound assessment of gestational age. Epidemiology, 6, 533-537. doi:10.1097/00001648-199509000-00012

[4] Sunjoh, F., Njamnshi, A.K., Tietche, F. and Kago, I. (2004) Assessment of gestational age in the Cameroonian newborn infant: A comparison of four scoring methods. Journal of Tropical Pediatrics, 50, 285-291. doi:10.1093/tropej/50.5.285

[5] Wingate, M.S., Alexander, G.R., Buekens, P. and Vahratian, A. (2007) Comparison of gestational age classifications: Date of last menstrual period vs. clinical estimate. Ann Epidemiology, 17, 425-430. doi:10.1016/j.annepidem.2007.01.035

[6] Darmstadt, G.L., Saha, S.K., Ahmed, A.S., Chowdhury, M.A., Law, P.A., Ahmed, S., et al. (2005) Effect of topical treatment with skin barrier-enhancing emollients on nosocomial infections in preterm infants in Bangladesh: A randomised controlled trial. Lancet, 365, 1039-1045.

[7] Darmstadt, G.L., Saha, S.K., Ahmed, A.S., Ahmed, S., Chowdhury, M.A., Law, P.A., et al. (2008) Effect of skin barrier therapy on neonatal mortality rates in preterm infants in Bangladesh: A randomized, controlled, clinical trial. Pediatrics, 121, 522-529. doi:10.1542/peds.2007-0213

[8] World Medical Association Declaration of Helsinki. (2000) Ethical principles for medical research involving human subjects. World Medical Association. http://www.wma.net/e/policy/17-c_e.html

[9] Naing, L., Winn, T. and Rusli, B.N. (2006) Practical issues in calculating the sample size for prevalence studies. Archives of Orofacial Sciences, 1, 9-14.

[10] Unicef (2005) The state of the world's children. New York.

[11] Basket, T. and Nagele, F. (2000) Nagele's rule: A reappraisal. British Journal of Obstetrics and Gynaecology, 107, 14-33.

[12] Dubowitz, L., Dubowitz, V. and Goldberg, C. (1970) Clinical assessment of gestational age in the newborn infant. Journal of Pediatrics, 77, 1-10. doi:10.1016/S0022-3476(70)80038-5

[13] Uche, N. (2007) Assessment and care of the newborn. In: Azubuike, J.C. and Nkanginieme, K.E., Eds., Pediatrics and Child Health in a Tropical Region, 2nd Edition, African Educational Services, Owerri, 163-177.

[14] Parkin, J.M., Hey, E.N. and Clowes, J.S. (1976) Rapid assessment of gestational age at birth. Archives of Disease in Childhood, 51, 259-263. doi:10.1136/adc.51.4.259

[15] Mittendorf, R., Williams, M.A. and Berkey, C.S. (1993) Predictors of human gestational length. American Journal of Obstetrics \& Gynecology, 168, 480. doi:10.1016/0002-9378(93)90476-Y

\section{ABBREVIATIONS}

GA: Gestational age;

NR: Naegle's rule;

DS: Dubowitz score;

CI: Confidence interval;

USS: Ultrasound scan;

SD: Standard deviation. 